\title{
O-Benzyl Xanthate Esters under Ni/Photoredox Dual Catalysis: Selective Radical Generation and $\mathrm{Csp}^{3}-\mathrm{Csp}^{2}$ Cross-Coupling
}

\author{
Brandon A. Vara, Niki R. Patel, and Gary A. Molander*(i)
}

Roy and Diana Vagelos Laboratories, Department of Chemistry, University of Pennsylvania, 231 South 34th Street, Philadelphia, Pennsylvania 19104-6323, United States

\section{Supporting Information}

ABSTRACT: Alkyl xanthate esters are perhaps best known for their use in deoxygenation chemistry. However, their use in cross-coupling chemistry has not been productive, which is due, in part, to inadequate xanthate activation strategies. Herein, we report the use of $O$-benzyl xanthate esters, readily derived from alcohols, as radical pronucleophiles in $\mathrm{Csp}^{3}-\mathrm{Csp}^{2}$ crosscouplings under $\mathrm{Ni}$ /photoredox dual catalysis. Xanthate ( $\mathrm{C}-$

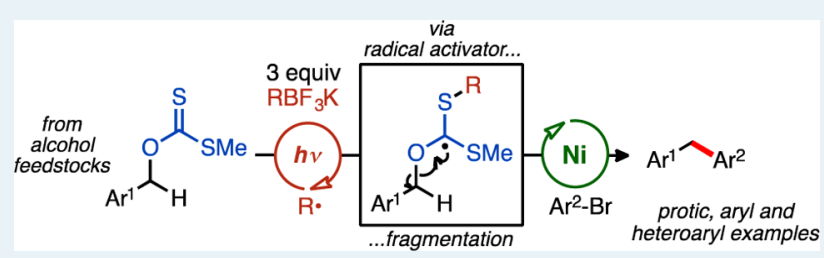

O) cleavage is found to be reliant on photogenerated (sec-butyl) radical activators to form new carbon-centered radicals primed for nickel-catalyzed cross-couplings. Mechanistic experiments support the fact that the key radical components are formed independently, and relative rates are carefully orchestrated, such that no cross reactivity is observed.

KEYWORDS: alkyl xanthate esters, cross-coupling, selective radical generation, carbon-centered radicals, metal-catalyzed reactions

$\mathrm{T}$ raditional two-electron, metal-catalyzed $\mathrm{Csp}^{3}-\mathrm{Csp}^{2}$ crosscoupling reactions provide efficient and selective access to architecturally rich molecules. However, challenges remain, with regard to accessing reliable and abundant alkyl nucleophiles for this class of coupling transformations. ${ }^{1}$ In particular, reactive alkylmetallic reagents $(\mathrm{RMgX}, \mathrm{RZnX}, \mathrm{RLi}$, etc.) have generally limited functional group tolerance, ${ }^{2}$ although advances in overcoming these barriers have been realized. ${ }^{3}$ More functional group-tolerant reagents, such as organoboron and organosilicon reagents, often lack the reactivity needed to overcome a rate-limiting transmetalation for effective cross-coupling.

The recent advent of $\mathrm{Ni} /$ photoredox dual catalysis by this group and others ${ }^{4}$ has surfaced as a complementary and mild alternative to traditional two-electron $\mathrm{Csp}^{3}-\mathrm{Csp}^{2}$ crosscoupling strategies, taking advantage of a relatively low-barrier interception of alkyl radicals by nickel catalysts, ${ }^{5}$ ultimately accessing high-valent $\mathrm{Ni}$ (III) intermediates. To date, substrate and functional group tolerance, under this single-electron crosscoupling paradigm, has been exceptional. ${ }^{6}$ In hopes of further expanding this synthetic toolbox and chemical space, novel radical precursors derived from abundant feedstock molecules (e.g., alkyl aldehydes ${ }^{7}$ and alkylsilanes ${ }^{8}$ ) have been targeted that are compatible under this dual catalytic manifold.

Alcohols remain one of the most abundant, naturally occurring organic synthons, and they carry with them enormous molecular diversity. Within cross-coupling contexts, select $\mathrm{sp}^{3}$-hybridized alcohol derivatives have been implemented as carbon electrophiles in low-valent, nickel-catalyzed reactions because of their inherent $\mathrm{C}-\mathrm{O}$ bond polarization, ${ }^{9}$ but have rarely functioned as precursors to nucleophilic entities. ${ }^{10}$ A compelling alternative to $\mathrm{C}-\mathrm{O}$ bond activation would be the ability to form carbon-centered radicals that might be parlayed toward new $\mathrm{C}-\mathrm{C}$ bond-forming reactions. Although the strong $\mathrm{C}-\mathrm{O} \sigma$ bond (bond dissociation energy (BDE) of $\sim 96 \mathrm{kcal} / \mathrm{mol}$ ) presents operational challenges, this strategy, if broadly applied, may offer advantages, particularly in accessing more available and diverse coupling partners.

O-Alkyl thiocarbonate (xanthate) esters $\left(\mathrm{R}^{1} \mathrm{OCSSR}^{2}\right)$ are attractive, bench-stable reagents in their ability to generate carbon radicals under Barton-McCombie-type conditions and are readily prepared in one step from a variety of alcohol feedstocks. ${ }^{11}$ Under these processes, $O$-alkyl xanthates are traditionally fragmented with an excess of a reducing agent (e.g., $\mathrm{HSnBu}_{3}$ ) and substoichiometric amounts of radical initiator (AIBN, peroxide, etc.) to prolong the radical chain event (e.g., Figure 1). A deoxygenation reaction employing specially tailored $O$-thiocarbamates $\left(E_{\text {red(average })} \approx-1.55 \mathrm{~V}\right.$ vs SCE) was recently reported, and these derivatives undergo a photocatalyzed, reductive single electron transfer (SET). ${ }^{12}$

Inspirational to this work, an alternative approach to the classic deoxygenation using $\mathrm{Bu}_{3} \mathrm{SnH}$ and $\mathrm{AIBN}$, for example, employs $\mathrm{BEt}_{3}$ and $\mathrm{O}_{2}$ as the initiator under otherwise relatively mild reaction conditions (benzene, $\mathrm{H}_{2} \mathrm{O}$, room temperature). ${ }^{13}$ The generated ethyl radical reacts with the xanthate ester, initiating a $\mathrm{C}-\mathrm{O}$ bond scission event (Figure 1) to produce a new carbon-centered radical that may be rapidly quenched by a hydrogen source, furnishing the reduced adduct. ${ }^{14}$ Since these pioneering studies, ethyl radical initiators (via $\mathrm{BEt}_{3} / \mathrm{O}_{2}$ ) have been widely adopted in various synthetic capacities, ${ }^{15}$ although experimental challenges are common. ${ }^{16}$

Received: March 9, 2017

Revised: April 30, 2017

Published: May 9, 2017 

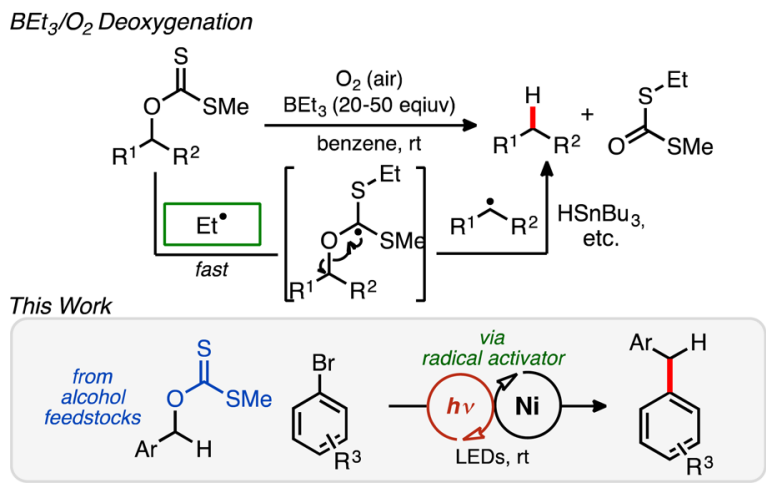

Figure 1. Radical cleavage of xanthate esters and application under $\mathrm{Ni} /$ photoredox dual catalysis.

We envisioned using carbon radical activators, generated mildly via SET photo-oxidation, to fragment suitable xanthate esters in cooperation with $\mathrm{Ni}$ catalysis, allowing access to new $\mathrm{Csp}^{3}-\mathrm{Csp}^{2}$ cross-coupled structures. This method would constitute an umpolung of reactivity, because alcohol derivatives in cross-coupling protocols are typically electrophilic (e.g., $\mathrm{OMs}, \mathrm{OMe}$ ), especially under Ni catalysis. ${ }^{9}$ To our knowledge, $O$-alkyl xanthate esters have never been employed as either electrophiles or nucleophiles in cross-coupling chemistry. ${ }^{17}$ In a broader sense, this approach may also validate photo-oxidizable radical precursors as alternatives to pyrophoric $\mathrm{BEt}_{3} / \mathrm{O}_{2}$ systems.

Conceptually, an excited-state photocatalyst ([PC]*; see Scheme 1) may be sufficiently oxidizing to generate an alkyl

Scheme 1. Proposed Ni/Photoredox Dual Catalytic CrossCoupling of $O$-Alkyl Xanthates Initiated by a Radical Activator

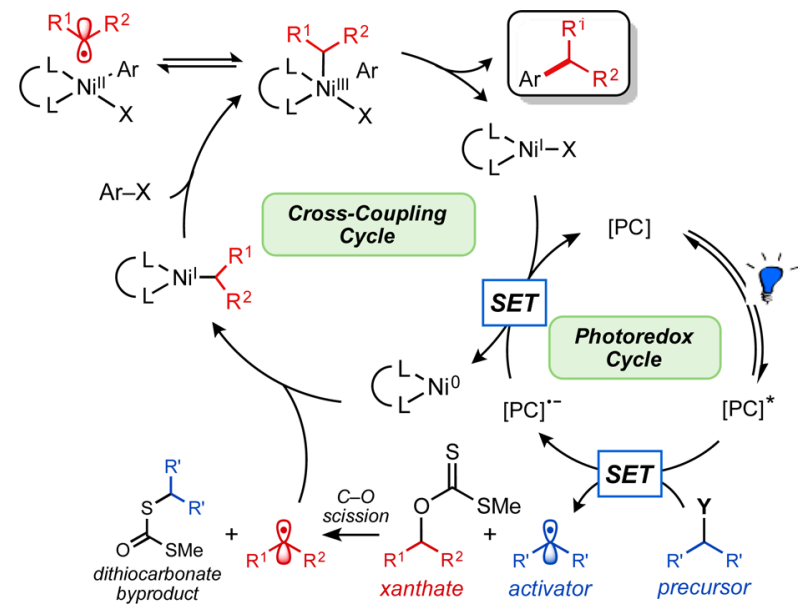

radical activator (blue) from an established radical precursor (e.g., $\mathrm{Y}=\mathrm{BF}_{3} \mathrm{~K}, \mathrm{CO}_{2} \mathrm{M}$, or ammonium silicate). This alkyl radical activator could rapidly react with the thiocarbonyl of the xanthate pronucleophile, initializing $\mathrm{C}-\mathrm{O}$ bond fragmentation. With subsequent xanthate cleavage and extrusion of dithiocarbonate, the alcohol-derived carbon radical (red), may enter the $\mathrm{Ni}$ coupling cycle, adding to $\mathrm{Ni}(0)$ as delineated in our previous studies. ${ }^{18}$ Following $\mathrm{Ni}(\mathrm{I})$ oxidative addition with the requisite aryl halide, a $\mathrm{Ni}$ (III) intermediate may be reached, producing the desired alkylated arene via reductive elimination. A SET event from the reduced form of the photocatalyst may regenerate $\mathrm{Ni}(0)$ from the $\mathrm{Ni}(\mathrm{I})$ species to close the intertwined dual catalytic cycles (see Scheme 1).

There were several evident challenges with the proposed reaction design. Radical hetero(homo)dimerization would result from multiple radicals simultaneously present, which is a challenge in multicomponent radical reactions. In addition, thiols and sulfides are well-known metal complexing agents, potentially inhibiting reaction progression through nickel sequestration. ${ }^{19}$ Perhaps most critically, the two radical components must have differentiable reactivity. The nucleophilic radical activator (blue) should be designed to react with the thiocarbonyl electrophile in preference to the nickel catalyst (e.g., eq 2 is desired over eq 3; see Scheme 2) to effect, first, the

Scheme 2. Potential Competing Transformations of Radical Components and $\mathrm{Ni}$ / Xanthate Affinity

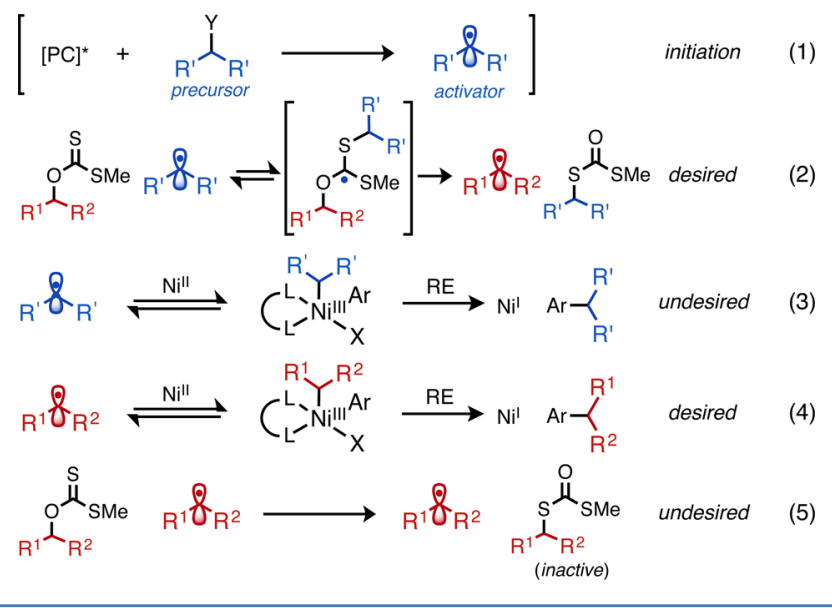

irreversible $\mathrm{C}-\mathrm{O}$ cleavage. The stoichiometric concentration of xanthate was anticipated to assist in this selectivity. The xanthate-derived radical (red) must then react smoothly with the nickel catalyst (eq 4 is desired over eq 5; see Scheme 2) with little to no cross reactivity. The potential advantage of using photoredox catalysis in this scenario is the ability to produce precise, substoichiometric quantities of reactive radical activator $(<2 \mathrm{~mol} \%)$, in contrast to radical chain processes, where the concentration of radicals is inherently capricious when using $\mathrm{O}_{2}$ as the initiator. ${ }^{16}$

Studies commenced with a wide variety of $O$-alkyl xanthate esters, including unactivated secondary/primary alkyl xanthates and activated secondary benzyl xanthates, with aryl bromide 2 . We observed promising reactivity with $O$-benzyl xanthate ester 1a, alongside the bench-stable $\left[\mathrm{Ni}(\mathrm{dtbbpy})\left(\mathrm{H}_{2} \mathrm{O}\right)_{4}\right] \mathrm{Cl}_{2}(4,5$ mol \%) precatalyst. ${ }^{7}$ Alkylammonium silicates, ${ }^{6,8}$, alkyltrifluor- $^{2}$ oborates, ${ }^{4 c, 6 a-c}$ and metal carboxylates ${ }^{4 d, 20}$ have been effective radical precursors, following SET oxidation under photoredox conditions, $^{21}$ and all were examined for their efficacy in xanthate (1a) C-O bond scission (Table 1; see the Supporting Information for complete studies). In these preliminary studies, low-molecular-weight alkyltrifluoroborates (labeled as $\mathbf{A}-\mathbf{E}$ in Table 1) proved to be the more efficacious radical initiators, using the photosensitizer $\left[\operatorname{Ir}\left\{\mathrm{dFCF}_{3} \mathrm{ppy}_{2}(\mathrm{bpy})\right] \mathrm{PF}_{6}\right.$ (5), with sec- $\mathrm{BuBF}_{3} \mathrm{~K}(\mathrm{~A})$, furnishing $3 \mathrm{a}$ in $94 \%$ yield (see entry 1 in Table 1) with no undesired radical couplings detected at room temperature. Cyclopropyltrifluoroborate (C) afforded no product, likely because of its greater s-character and higher oxidation potential. ${ }^{6 a}$ Organic and inorganic bases were found to be a hindrance to reaction progression under various 
Table 1. Deviations from Standard Reaction Conditions Employing Xanthate 1a under Ni/Photoredox Dual Catalysis $^{a}$

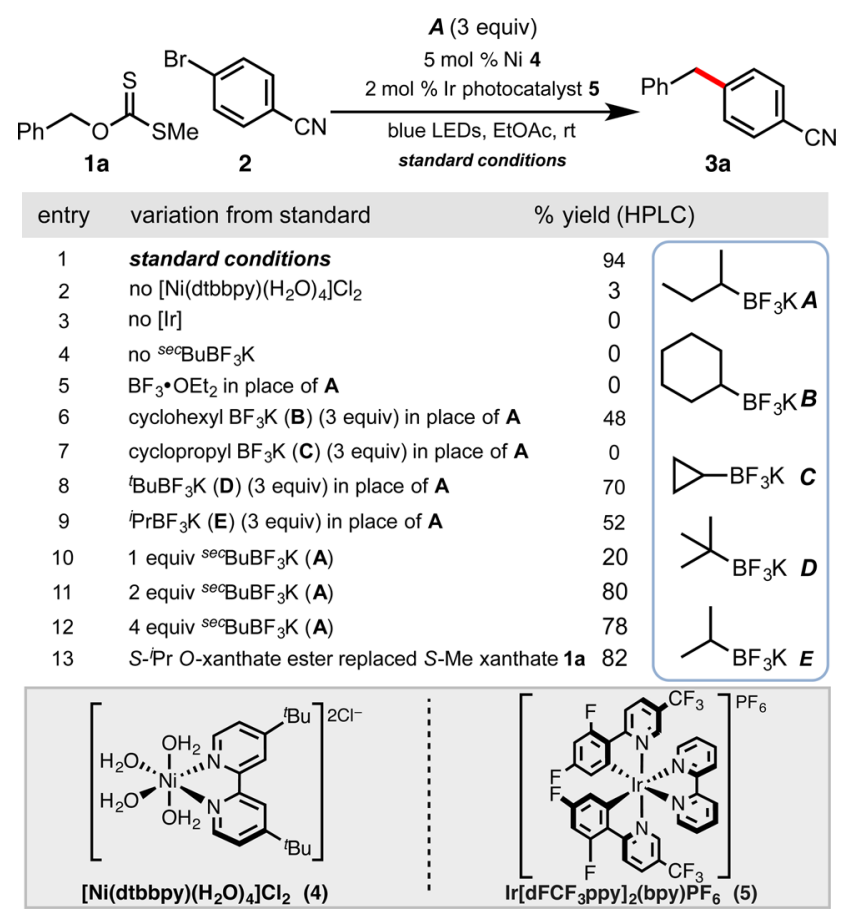

${ }^{a}$ Reactions performed on $0.1 \mathrm{mmol}$ scale for $48 \mathrm{~h}$, unless otherwise noted. HPLC yields reported as compared to internal standards. See the Supporting Information for additional details.

conditions examined. Control experiments revealed that lightemitting diodes (LEDs), iridium, and nickel catalysts were all necessary components for this transformation (see entries 2-4 in Table 1). Three equivalents of the alkyltrifluoroborate is deemed necessary, and all sec- $\mathrm{BuBF}_{3} \mathrm{~K}$ is consumed in the reaction, as determined by ${ }^{19} \mathrm{~F}$ NMR monitoring (see the Supporting Information). Comprehensively quantifying the fate of the reagent could not be fully ascertained. Regardless, the use of the pyrophoric $\mathrm{BEt}_{3}$ reagent may be replaced with a benchstable solid.

With satisfactory conditions in hand to promote the coupling of benzyl $O$-xanthate esters, an array of xanthates (prepared using base, $\mathrm{CS}_{2}, \mathrm{MeI}$, and used without purification) were examined with bromide 2 (see Scheme 3). Electron-rich and electron-deficient $O$-benzyl xanthates are similarly welltolerated under the conditions. Oxygenated arenes (3b, 3c, $3 \mathbf{j})$, including free phenol $(3 \mathbf{h})$, are perhaps most compatible under the developed dual catalytic conditions. Heteroaromatic, methanol-derived xanthates are easily prepared and employed, yielding pyridines $(\mathbf{3 g})$, furans $(\mathbf{3} \mathbf{j})$, and thiophenes $(3 \mathbf{l})$. These base-free coupling conditions also tolerate protic functional groups (3f, $3 \mathbf{h})$, where many analogous, benzyl-derived pronucleophiles (e.g., $\mathrm{BnBF}_{3} \mathrm{~K}$ or $\mathrm{BnZnCl}$, derived from benzyl halides), in contrast, would be difficult to prepare directly in their unprotected form. Secondary benzyl and unactivated alkyl xanthates (Chugaev elimination product could be observed) ${ }^{22}$ were not well-tolerated in these studies, even upon mild heating, and often resulted in a mixture of products.

Numerous aryl bromides were examined next, alongside an O-benzyl xanthate ester under the optimized conditions (Scheme 3). Electron-deficient aryl bromides and heteroaryl bromides are quite compatible, although electron-rich (e.g., 3s)
Scheme 3. O-Benzyl Xanthate and Aryl Halide Scope ${ }^{a}$

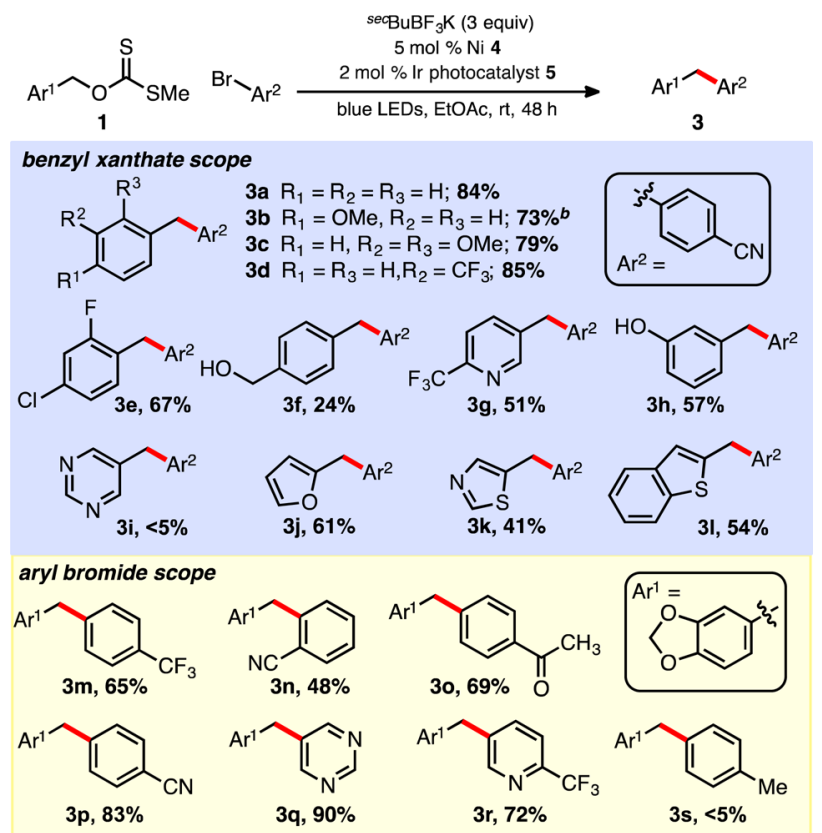

${ }^{a}$ Conditions are as described in Table 1 (entry 1). $0.5 \mathrm{mmol}$ scale reactions; isolated yields are reported. ${ }^{b}$ Two equivalents of ${ }^{s e c} \mathrm{BuBF}_{3} \mathrm{~K}$ employed.

and even electron-neutral bromides are largely not as robust. Functional handles such as ketones (3o) and nitriles (3n, 3p) are also well tolerated. Pyridines (3r) and a pyrimidine (3q) can be prepared in moderate to excellent yields $(72 \%-90 \%)$.

To showcase the potential utility of benzyl alcohol-derived pronucleophiles, we explored a bidirectional synthesis of 9 from 4-bromobenzyl alcohol (7), using sequential $\mathrm{Ni} /$ photoredox dual catalyzed reactions (Scheme 4). Bicycloheptylsilicate 6,

Scheme 4. Rapid, Bidirectional Coupling Affords 9 under $\mathrm{Ni}$ /Photoredox Dual Catalysis ${ }^{a}$

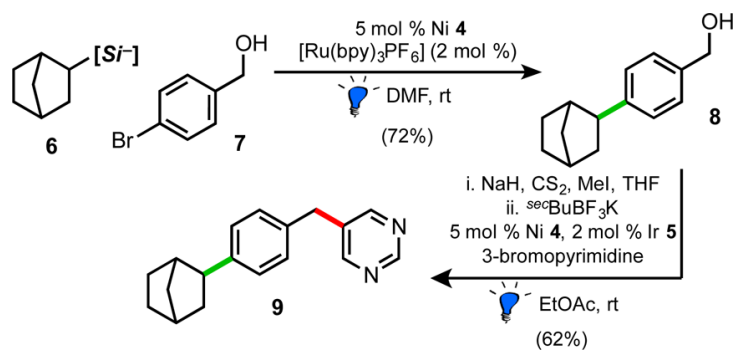

${ }^{a}$ Isolated yields. $\left[\mathrm{Si}^{-}\right]=b i s($ catechol $)$ ammonium silicate.

which is a secondary alkyl radical precursor, ${ }^{8}$ was coupled efficiently with 7 under mild conditions, yielding intermediate 8. Benzyl alcohol 8 underwent xanthate activation, and following workup and solvent exchange, reacted smoothly with 3-bromopyrimidine to furnish the final dialkylated product 9 in $62 \%$ yield over the two-step sequence.

Additional experiments aided elucidation of the mechanistic nuances of this reaction, and data collected generally support the proposed dual-catalyzed mechanism. Remarkably, the undesired sec-butyl arene cross-coupled product $\mathbf{1 0}$ was never observed throughout these studies when xanthate was present (Figure 2). However, in the absence of the xanthate ester under 


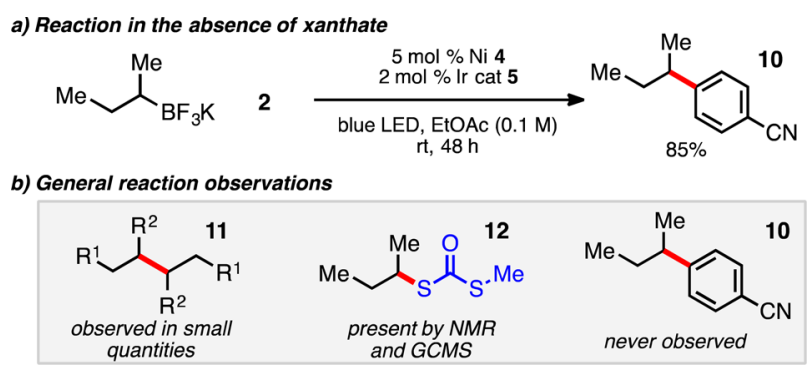

Figure 2. Mechanistic studies and observations.

otherwise standard reaction conditions, full conversion to $\mathbf{1 0}$ can be observed, ${ }^{6 a, 24}$ critically demonstrating the rapid nature of sec-butyl radical addition to the xanthate thiocarbonyl and the ability to control reaction outcomes based on relative rates as initially designed (Figure 2a). Furthermore, homodimerized radical activator 11 can be observed in the reaction, ${ }^{23}$ in addition to the dialkyl dithiocarbonate $\mathbf{1 2}$ byproduct that is generated, validating the notion that sec-butyl radical is key to reaction initiation. Studies also revealed that external addition of dialkyl dithiocarbonate 12 ( 0.7 equiv) to the reaction decreased the reaction rate, presumably through nickel coordination. $^{25}$

In conclusion, we present the first example of $O$-benzyl xanthate ester pronucleophiles derived from abundant benzyl alcohols in $\mathrm{Csp}^{3}-\mathrm{Csp}^{2}$ cross-coupling reactions under photoredox/Ni dual catalysis, which is reliant on the unique generation of radical activators to promote xanthate cleavage. The mild reaction conditions employ low-molecular-weight, commercially available potassium alkyltrifluoroborates as radical precursors for xanthate $\mathrm{C}-\mathrm{O}$ bond scission under base-free conditions. The relative rates of two distinct radical components are judiciously controlled in the reaction, capitalizing on the inherent selectivity of photoredox catalysis. The development of a versatile new functional group other than halide derivatives adds an important new dimension to $\mathrm{Csp}^{3}-$ Csp ${ }^{2}$ cross-coupling chemistry.

\section{ASSOCIATED CONTENT}

\section{S Supporting Information}

The Supporting Information is available free of charge on the ACS Publications website at DOI: 10.1021/acscatal.7b00772.

Experimental details and spectral data (PDF)

\section{AUTHOR INFORMATION}

\section{Corresponding Author}

*Tel.: (215) 573-8604. Fax: (215) 573-7165. E-mail: gmolandr@sas.upenn.edu.

\section{ORCID}

Gary A. Molander: 0000-0002-9114-5584

\section{Funding}

We thank NIGMS (No. R01 GM113878) for financial support of this research.

\section{Notes}

The authors declare no competing financial interest.

\section{ACKNOWLEDGMENTS}

Dr. Charles Ross, III (University of Pennsylvania), is acknowledged for collection of HRMS data; Sigma-Aldrich is acknowledged for the kind donation of $\mathrm{IrCl}_{3}$; and Dr. Álvaro
Gutiérrez-Bonet is acknowledged for the preparation of $\left[\mathrm{Ni}(\mathrm{dtbbpy})\left(\mathrm{H}_{2} \mathrm{O}\right)_{4}\right] \mathrm{Cl}_{2}$. David Primer (University of Pennsylvania) and John Tellis (Genentech) are thanked for insightful contributing discussions. Dr. Simon Lang (University of Pennsylvania) is thanked for additional data collection.

\section{REFERENCES}

(1) Hartwig, J. F. Organotransition Metal Chemistry: From Bonding to Catalysis; University Science: Sausalito, CA, 2010.

(2) Rudolph, A.; Lautens, M. Angew. Chem., Int. Ed. 2009, 48, 2656.

(3) (a) Benischke, A. D.; Knoll, I.; Rérat, A.; Gosmini, C.; Knochel, P. Chem. Commun. 2016, 52, 3171. (b) Toriyama, F.; Cornella, J.; Wimmer, L.; Chen, T.-G.; Dixon, D. D.; Creech, G.; Baran, P. S. J. Am. Chem. Soc. 2016, 138, 11132. For a review, see: (c) Haas, D.; Hammann, J. M.; Greiner, R.; Knochel, P. ACS Catal. 2016, 6, 1540 and references therein.

(4) Seminal report: (a) Kalyani, D.; McMurtrey, K. B.; Neufeldt, S. R.; Sanford, M. S. J. Am. Chem. Soc. 2011, 133, 18566. (b) Ye, Y.; Sanford, M. S. J. Am. Chem. Soc. 2012, 134, 9034. (c) Tellis, J. C.; Primer, D. N.; Molander, G. A. Science 2014, 345, 433. (d) Zuo, Z. W.; Ahneman, D. T.; Chu, L. L.; Terrett, J. A.; Doyle, A. G.; MacMillan, D. W. C. Science 2014, 345, 437.

(5) Examples with transition metals other than nickel have been reported: (a) Sahoo, B.; Hopkinson, M. N.; Glorius, F. J. Am. Chem. Soc. 2013, 135, 5505. (b) Hopkinson, M. N.; Sahoo, B.; Li, J.-L.; Glorius, F. Chem.-Eur. J. 2014, 20, 3874.

(6) (a) Primer, D. N.; Karakaya, I.; Tellis, J. C.; Molander, G. A. J. Am. Chem. Soc. 2015, 137, 2195. (b) Karakaya, I.; Primer, D. N.; Molander, G. A. Org. Lett. 2015, 17, 3294. (c) Yamashita, Y.; Tellis, J. C.; Molander, G. A. Proc. Natl. Acad. Sci. U. S. A. 2015, 112, 12026. (d) Tellis, J. C.; Amani, J.; Molander, G. A. Org. Lett. 2016, 18, 2994. (e) Karimi-Nami, R.; Tellis, J. C.; Molander, G. A. Org. Lett. 2016, 18, 2572. (f) Patel, N. R.; Molander, G. A. J. Org. Chem. 2016, 81, 7271. (g) Vara, B. A.; Jouffroy, M.; Molander, G. A. Chem. Sci. 2017, 8, 530. (7) Gutiérrez-Bonet, A.; Tellis, J. C.; Matsui, J. K.; Vara, B. A.; Molander, G. A. ACS Catal. 2016, 6, 8004.

(8) Jouffroy, M.; Primer, D. N.; Molander, G. A. J. Am. Chem. Soc. 2016, 138, 475 .

(9) (a) Kuwano, R.; Kondo, Y. Org. Lett. 2004, 6, 3545. (b) Guan, B. T.; Xiang, S. K.; Wang, B. Q.; Sun, Z. P.; Wang, Y.; Zhao, K. Q.; Shi, Z. J. J. Am. Chem. Soc. 2008, 130, 3268. For enantioselective and stereospecific variants, see: (c) Taylor, B. L. H.; Swift, E. C.; Waetzig, J. D.; Jarvo, E. R. J. Am. Chem. Soc. 2011, 133, 389. (d) Harris, M. R.; Hanna, L. E.; Greene, M. A.; Moore, C. E.; Jarvo, E. R. J. Am. Chem. Soc. 2013, 135, 3303.

(10) While this research was underway, a Csp ${ }^{3}-\mathrm{Csp}^{2}$ cross-coupling of redox-active, alkyl metal oxalates (radical pronucleophiles) was reported with various alcohol partners: Zhang, X.; MacMillan, D. W. C. J. Am. Chem. Soc. 2016, 138, 13862.

(11) For pioneering reports, see: (a) Barton, D. H. R.; McCombie, S. W. J. Chem. Soc., Perkin Trans. 1 1975, 1574. (b) Barrett, A. G. M.; Prokopiou, P. A.; Barton, D. H. R. J. Chem. Soc., Perkin Trans. 1 1981, 1510. (c) Barton, D. H. R.; Crich, D.; Löbberding, A.; Zard, S. Z. Tetrahedron 1986, 42, 2329. (d) For a review on thiocarbonyls in radical chemistry, see: Crich, D.; Quintero, L. Chem. Rev. 1989, 89, 1413.

(12) Traditional $O$-alkyl xanthates have very low reduction potentials, as was confirmed by our CV studies: $E_{\mathrm{ox}}=+1.75 \mathrm{~V}$. For a report of photoredox-active dithiocarbamates, see: Chenneberg, L.; Baralle, A.; Daniel, M.; Fensterbank, L.; Goddard, J.-P.; Ollivier, C. Adv. Synth. Catal. 2014, 356, 2756.

(13) Initial reports; see: (a) Nozaki, K.; Oshima, K.; Utimoto, K. Tetrahedron Lett. 1988, 29, 6125. (b) Barton, D. H. R.; Ok, J. D.; Jaszberenyi, J. C. Tetrahedron Lett. 1990, 31, 3991. Also see: Spiegel, D. A.; Wiberg, K. B.; Schacherer, L. N.; Medeiros, M. R.; Wood, J. L. J. Am. Chem. Soc. 2005, 127, 12513.

(14) For a report on $\mathrm{Et}_{3} \mathrm{~B} /$ air reaction kinetics and mechanism, see: Jin, J.; Newcomb, M. J. Org. Chem. 2007, 72, 5098. 
(15) For a review and references therein, see: (a) Ollivier, C.; Renaud, P. Chem. Rev. 2001, 101, 3415. (b) N-Heterocyclic carbene boryl radicals; see: Ueng, S.-H.; Solovyev, A.; Yuan, X.; Geib, S. J.; Fensterbank, L.; Lacôte, E.; Malacria, M.; Newcomb, M.; Walton, J. C.; Curran, D. P. J. Am. Chem. Soc. 2009, 131, 11256.

(16) Curran, D. P.; McFadden, T. R. J. Am. Chem. Soc. 2016, 138, 7741.

(17) $\mathrm{Fu}$ and coworkers briefly explored xanthate electrophiles; see: Oelke, A. J.; Sun, J.; Fu, G. C. J. Am. Chem. Soc. 2012, 134, 2966.

(18) Gutierrez, O.; Tellis, J. C.; Primer, D. N.; Molander, G. A.; Kozlowski, M. C. J. Am. Chem. Soc. 2015, 137, 4896.

(19) Argyle, M. D.; Bartholomew, C. H. Catalysts 2015, 5, 145.

(20) (a) Johnston, C. P.; Smith, R. T.; Allmendinger, S.; MacMillan, D. W. C. Nature 2016, 536, 322. (b) Zuo, Z.; Cong, H.; Li, W.; Choi, J.; Fu, G. C.; MacMillan, D. W. C. J. Am. Chem. Soc. 2016, 138, 1832. (21) Tellis, J. C.; Kelly, C. B.; Primer, D. N.; Jouffroy, M.; Patel, N. R.; Molander, G. A. Acc. Chem. Res. 2016, 49, 1429.

(22) Benkeser, R. A.; Hazdra, J. J. J. Am. Chem. Soc. 1959, 81, 228.

(23) $1,1^{\prime}-\mathrm{Bi}$ (cyclohexane) dimer can be observed by GCMS when activator B (Table 1) was employed.

(24) Spectral confirmation of $\mathbf{1 0}$ is confirmed: Liu, Z.; Dong, N.; Xu, M.; Sun, Z.; Tu, T. J. Org. Chem. 2013, 78, 7436.

(25) Byproduct 12 was added to otherwise normal reaction conditions and suppressed product formation (see the Supporting Information for a complete study). 